\title{
Deformation Behaviour of Tunnels under Different Loading Conditions: State of Art
}

\author{
Parvesh Kumar ${ }^{1}$, Amit Kumar Shrivastava ${ }^{1}$ \\ ${ }^{1}$ Civil Engineering Department, Delhi Technological University \\ Bhawana Road, Delhi, India \\ parveshkumar_2k16phdce06@dtu.ac.in; aksrivastava@dce.ac.in
}

\begin{abstract}
Construction of tunnels as transport medium has become a very important part in various civil engineering practises. Tunnels are constructed and commonly being used for different purposes such as for underground mining, underground parking facilities, military purposes, underground gas pipelines, transportation purposes in metro cities and many more fields. In the present study, the deformation behaviour of tunnels under various loading conditions that may be static load acting on the tunnels due to the overburden pressure, dynamic load due to the earthquakes and impact load causes due to terrorist activities is studied. Various factors which may be responsible for causing deformation cracks in tunnels are being studied. Various theories on stress distribution in tunnels are given by a lot of researchers were also studied.
\end{abstract}

Keywords: Deformation, Underground Structures, Cover depth, In-situ stress.

\section{Introduction}

Tunnels are classified as underground artificial passages which are constructed without making any disturbance in the ground surface. Tunnels are generally used for various purposes in our day to day life. In4000 B.C underground caves were made by human being for their shelter. Therefore, contraction of tunnels and underground passages is very old activity which is commonly performed by human being for their different purposes. Tunnels can be constructed through rock masses, hills, rivers etc. In the present day, tunnels are used for various purposes. Apart from this, tunnels can be used for different application work which is related to various civil and military fields. Tunnels are used as a means of transportation in hilly areas where modes of transportations are very less or negligible in some places. Underground structures are used for construction of railways lines, roads etc. Also, they are used for various military practices. In difficult conditions, such as soft ground, large jointed rocks, massive hills, underground structures are very effective. When we talk about the underground structures it is very important to understand the distributions of various stresses on tunnels. There are various types of loads which are subjected on the tunnels structures. Due to these loads subjected over tunnels, deformation occurs which further effects the stability of the structures. Generally, the underground structures are subjected to static load, dynamic loads and impact loads. Static load is caused due to the overburden pressure acting on the structure whereas dynamic and impact load is caused in earthquake and blast conditions. Stability of underground structures is very important aspect which should be kept in mind while designing the structure. In unstable conditions, the structure may deform in a very short span of time. When the tunnels are subjected to static loading conditions, the maximum load occurs at the roof and the side walls of the tunnels structures. If the structure is not reinforced properly then the structure may subjected to tensile and compression failure. Various theories were given by a lot of researchers to study the stress distribution in both single and twin tunnels. From these theories it may be noted that the tunnel structures are basically subjected to three types of stresses i.e. radial stress, tangential stress and shear stress. Different equations of stresses are derived by various researchers which are discussed briefly in this paper.

\section{Deformations of tunnels under different conditions}

The influence of the various factors on the deformation behavior of tunnels has been studied by many researchers. From the review it can be noticed that there are a lot of factors which affect the stability of underground structures. Some of them are summarized here. Stability of tunnels mainly depends upon the relative position of tunnel and its construction procedure. Chehade and Shahrour, (2008) studied the interaction between the twin tunnels with the help of numerical software. The 
studied was done to know how the position of the twin tunnels and their construction procedure may affect the settlement behavior of the soil structures. For the study, three different alignments of twin tunnels were selected i.e. horizontally, vertically and inclined. From the results, it can be concluded that when the upper tunnel is constructed first, it may lead to higher settlement as compare to that obtained by construction of lower tunnel at first. It was also noted that when the tunnels are vertically aligned, they may cause the highest settlement of soil. Idris et al., (2008), conducted a study to determine the deformation behavior of ancient tunnels structure by using UDEC. The studied was done to determine the effect of various factors such as masonry block cohesion, tensile strength and friction angle. From the study, it was notified that there is a significant effect of cohesion and friction angle on the masonry behavior. Jia and Tang, (2008) conducted a study on the failure mechanism of tunnel in jointed rock mass using numerical code RFPA. The study deals with the influence of different dip angles of layered joint on the stability of the tunnels. The study indicates that both the dip angle and lateral pressure have significant impact on the failure mode. As the dip angle of the joint increases the displacement of the sidewall also increases. Nunes and Meguid, (2009) conducted an experimental study to determine the effect of stresses caused due to the overlying soil on the stability behavior of tunnel. Experimental tests were performed to know the influence of the overlying layer on tunnels deformation behavior. Effect of stiffness ratio and effect of layer thickness is also studied. Numerical investigation was also done by using Plaxis V8 software to compare the results obtained from experimental tests. From results, it can be notified that there is a significant effect on the stresses acting on tunnel due to the presence of the soil layer above the tunnel. It was concluded that if the thickness and location of the soil layer which is present above the tunnel changes, then the stress distribution may also vary. Idris et al., (2009) conducted a study on the numerical modeling of masonry joints degradation in built tunnels. In this study an attempt is made to study the influence of the mechanical properties of the masonry joints on the tunnel behavior by the help of experimental design method distinct elements numerical modeling. The factors selected for this study were masonry joint, cohesion, joint tensile strength and joint friction angle. It was concluded that the joint cohesion is the most important factor followed by joint tensile strength and finally the joint friction angle. Elshamy et al., (2013) conducted a study to determine the deformation behavior of different shapes of twin tunnels in soft clay. A numerical model is constructed as a plane strain problem taking into consideration the soil-structure interaction. The study was done to examine the effect of thickness-radius ratio on the tunnel deformation behavior, forces acting on the tunnel and the stress distribution. The settlement of the surface was noticed for various shapes and dimension of different twin tunnels. Three different tunnel shapes (circular, elliptical and rectangle with circular arch) were selected for this study. From the results, it was concluded that the circular shape tunnel is the best twin tunnel as vertical displacement and internal forces acting on the tunnel structure were less in circular tunnels as compare to the other two shapes of twin tunnels. It was also noticed that there is an increase in the internal forces up to $40 \%$, when the diameter of the tunnel varies from 8 to $12 \mathrm{~m}$. Also, there is an increase in soil stresses and surface displacement when the diameter is increased from 8 to 12 meters.

Zhu et al., (2015) made an attempt to study the effect of principle stress orientation on the stability of regular tunnel and crack tunnel. For that, three typical tunnel models were prepared and the materials used for tunnel model formation were cement mortar and sandstone. The first one was a regular tunnel model loaded by various orientation principle stresses. The second one was a tunnel with various inclination cracks. And the third one is a tunnel with a fixed angle crack loaded by various orientation principle stresses. A $5 \mathrm{~cm}$ radial crack with different inclination angles $\beta$ at tunnel spandrels is made. The results show that the critical compressive stress varies with the angle of inclination of the crack. When $\beta$ increases from $0^{\circ}$ to $135^{\circ}$, the critical stress decreases and when $\beta$ is about $135^{\circ}$ the critical stress is lowest. As an increase in the angle of inclination $\beta$ from $135^{\circ}$ to $180^{\circ}$, the critical stress increases. Khan et al., (2015) conducted a study to analyze the interactive behavior between tunnel lining and rock with the variation of stresses and deformation. In this study different tunnels section i.e. circular; semicircular and horse shoe were considered in both fractured and unfractured rock. Stress and deformation are analyzed in all the three section and the most suitable section is determined. Numerical modeling using FEM (Abacus Software) is done for modeling and meshing of tunnel in 2D. From results it was concluded that for unfractured rock the most suitable section from vertical stress point of view is horse shoe and from deformation point of view is semicircular section. For fractured rock the most suitable section from both points of view is semicircular section. Mishra et al., (2016) conducted a study to determine the deformation behavior of shallow tunnels under static and dynamic loading. For this study ABACUS is used as modeling software which is based on FEM numerical tool. In this study natural soft rock conditions are 
simulated in laboratory by using Plaster of Paris and mix material as tunnel model material. Then the results which are obtained from numerical modeling are then compared with experimental results. From the results, it was noticed that in case of static loading the length of the crack is large as compare to the crack obtained in case of dynamic loading. It was noticed that the zone is totally crushed in case of dynamic loading. Tiwari et al., (2016) conducted a study on analyze the behavior of twin tunnel under dynamic conditions. The twin tunnel consists of tunnel reinforced concrete lining which is subjected to internal blast loading. Numerical modeling is done with the help of ABACUS. Pressure acting in the Reinforced concrete lining and the surrounding soil mass, which is caused by the explosive induced shock wave, was determined for both tunnels. And from the results, it was concluded that the damage and deformation of reinforced concrete lining and soil mass depend upon charge weight and the clearance between the tunnels. Ahmad and Bahador, (2016) conducted a study to analyze the effect of different factors on settlement behavior of ground due to excavation of twin tunnels. Comparison of Flac2D and Flac3D modeling was done. The results concluded that the 3D model show a more realistic profile than 2D model. The maximum settlement was occurred along the center line of the tunnel at ground level and the highest rate of displacement in the model occurred in the crown of the tunnel. It was also observed that the impact area of tunnel excavation is extended up to 4 times the diameter from the center of the tunnel after that there is no change. It was also noticed that there was larger displacement in case of simultaneous excavation this may be due to the interaction between the tunnel faces. Kumar and Shrivastava, (2017) reviewed the various factors which effect the stability of underground structures. It was concluded that the stability of tunnels depends upon many factors such as types of tunnel section, crack inclination angle and stress caused on tunnels due to overlying strata. Shalabi, (2017) conducted a study on the interaction behavior of shallow twin tunnels of circular shape by using FE analysis. The study was conducted to determine the effect on the stability behavior of tunnel when the spacing between the tunnels varies. From the results it was noticed that deformation effect will be more when the spacing between the tunnels is less. Mishra, (2017) conducted a study to determine the effects of static and cyclic loading conditions on lined and unlined tunnels samples. From the results it was concluded that in case of unlined tunnels the number of cycles required to damage the tunnel is less from lined samples this is due to the resistance offered by the lining material in case of lined samples. Muhammad, (2017) conducted a study on circular tunnels under static and dynamic loads at different depths. Numerical investigation is conducted with the help of a finite element software tool MIDAS GTS NX. It was noticed that the depth of the tunnel has a significant effect on the stability behavior.

\section{Various Theories on stress distribution in Tunnels}

During the designing of the tunnel, evaluation of stresses and displacement around the circular opening is primary requirement. There are various methods are available which are helpful in determing the stress distribution and displacement behaviour of tunnels when they are subjected to different loading conditions. Numerous solutions have been given for the distribution of stresses around the circular opening. A lot of researchers had giver their theories and solutions for the stresses acting around the underground structures. Kirsch was the first who had given the analytical solution for the stress and displacement around the circular opening in plain strain conditions in 1898. According to Kirsch, for a circular opening of radius "a" and radial distance " $r$ ", the expression for radial, tangential and shear stress are given.

$$
\begin{gathered}
\sigma_{\mathrm{r}}=1 / 2 \cdot\left(\sigma_{\mathrm{x}}+\sigma_{\mathrm{z}}\right) \cdot\left(1-\mathrm{a}^{2} / \mathrm{r}^{2}\right)+1 / 2 \cdot\left(\sigma_{\mathrm{x}}-\sigma_{\mathrm{z}}\right) \cdot\left(1+3 \mathrm{a}^{4} / \mathrm{r}^{4}-4 \mathrm{a}^{2} / \mathrm{r}^{2}\right) \cdot \cos 2 \theta \\
\sigma_{\theta}=1 / 2 \cdot\left(\sigma_{\mathrm{x}}+\sigma_{\mathrm{z}}\right) \cdot\left(1+\mathrm{a}^{2} / \mathrm{r}^{2}\right)-1 / 2 \cdot\left(\sigma_{\mathrm{x}}-\sigma_{\mathrm{z}}\right) \cdot\left(1+3 \mathrm{a}^{4} / \mathrm{r}^{4}\right) \cdot \cos 2 \theta \\
\tau_{\mathrm{r} \theta}=-1 / 2 \cdot\left(\sigma_{\mathrm{x}}-\sigma_{\mathrm{z}}\right) \cdot\left(1-3 \mathrm{a}^{4} / \mathrm{r}^{4}+2 \mathrm{a}^{2} / \mathrm{r}^{2}\right) \cdot \sin 2 \theta
\end{gathered}
$$

Where, $\sigma_{\mathrm{r}}=$ radial stress

$\sigma_{\theta}=$ tangential stress

$\tau_{\mathrm{r} \theta}=$ shear stress

$\sigma_{\mathrm{z}}=\gamma \cdot \mathrm{h}=$ overburden pressure

$\sigma_{\mathrm{x}}=\mathrm{K} \cdot \sigma_{\mathrm{z}}=$ horizontal pressure

$\mathrm{a}=$ radius of the opening

$\mathrm{r}=$ radial distance from the centre of opening 
From the above equations given by Kirsch (1898) following conclusions can be drawn:

(i) In case $\sigma_{x}=0$, the maximum tangential stresses will be equal to three times the applied stresses.

(ii) For $\sigma_{\mathrm{x}}=0$, tangential stress will be equal to the applied stress with opposite sign at the boundary of the opening when $\theta=90^{\circ}$ or $270^{\circ}$.

(iii) For $\sigma_{\mathrm{x}}=\sigma_{\mathrm{z}}$, the maximum tangential stress will be equal to the twice the applied stress. Also the maximum tangential stress will occur at the boundary of circular opening.

Terzaghi and Richart (1952) also gave some equation to study the stress distribution behaviour of circular opening by using Kirsch solution. The equations given by Terzaghi and Richart (1952) are as follows:

$$
\begin{gathered}
\sigma_{\mathrm{h}}=1 / 2 \cdot\left(\sigma_{\theta}+\sigma_{\mathrm{r}}\right)+1 / 2\left(\sigma_{\theta}-\sigma_{\mathrm{r}}\right) \cdot \cos 2 \theta+\tau_{\mathrm{r} \theta} \sin 2 \theta \\
\sigma_{\mathrm{v}}=1 / 2 \cdot\left(\sigma_{\theta}+\sigma_{\mathrm{r}}\right)-1 / 2\left(\sigma_{\theta}-\sigma_{\mathrm{r}}\right) \cdot \cos 2 \theta-\tau_{\mathrm{r} \theta} \sin 2 \theta \\
\tau_{\mathrm{vh}}=-1 / 2 \cdot\left(\sigma_{\theta}-\sigma_{\mathrm{r}}\right) \sin 2 \theta+\tau_{\mathrm{r} \theta} \cos 2 \theta
\end{gathered}
$$

Where, $\sigma_{\mathrm{r}}=$ radial stress

$\sigma_{\theta}=$ tangential stress

$\tau_{\mathrm{r} \theta}=$ shear stress

$\sigma_{\mathrm{z}}=\gamma \cdot \mathrm{h}=$ overburden pressure

$\sigma_{\mathrm{x}}=\mathrm{K} \cdot \sigma_{\mathrm{z}}=$ horizontal pressure

$\mathrm{a}=$ radius of the opening

$r=$ radial distance from the centre of opening

\section{Conclusion}

From the above review following conclusions can be made:

(i) There are many factors which affect stability of underground structures such as type of soil, overburden pressure, rock profile, discontinuities or joints present in rock, relative position of tunnels, construction procedure and alignment of the tunnel etc.

(ii) There is a significant effect of the shape of the tunnel section on its stability behaviour. It can be noticed from the above review that circular section is best shape tunnel section as the deformation experienced in the circular section is least as compare to other tunnel sections.

(iii) Various stress distribution theories are derived by different researchers and it can be noticed that stress distribution behaviour of tunnel is mainly affected by radial stresses, tangential stresses and shear stresses. Also, with the help of these theories the boundary conditions of the tunnel model can also be decided.

\section{Acknowledgements}

We would like to convey our deep gratitude to Head of the Civil engineering Department and all the faculty members for their cooperation during the study. We would also like to thank Delhi Technological University authorities for their sponsorship and financial support. The helpful and supportive behavior of laboratory assistants of rock mechanics lab and geotechnical engineering lab is appreciatively acknowledged.

\section{References}

[1] E. A. Elshamy, G. Attai, H. Fawzy and K. Abdel, "Behaviour of different shap of twin tunnels in soft clay soil," Int. J. Engineering and Innovative Technology (IJEIT), vol. 2, 2013. 
[2] F. Ahmad and Y. Bahodor, "Analysis of the impact of various factors on the ground settlement profile due to excavation twin tunnels," International Conference in New Research on Civil Architectural and Urban management,2016.

[3] F. Chehade and I. Shahrour, "Numerical analysis of the interaction between twin-tunnels: Influence of the relative position and construction procedure," Tunnelling and Underground Space Technology, vol. 23, pp. 210-214, 2008.

[4] F. Shalabi, "Interaction of Twin Circular shallow tunnels in soil-Parametric Study," Open Journal of Civil Engineering, vol. 7, pp.100-115, 2017.

[5] I. A. Khan, K. Venkatesh, R. K. Srivastava, "Fractured and Un-fractured analysis of a rock tunnel by finite element method," Indian Geotechnical Conference, 2015.

[6] J. Idris, T. Verdel and M. Al-Heib, "Numerical modelling and mechanical behaviour analysis of ancient tunnel masonry structures," Tunnelling and Underground Space Technology, vol. 23, pp. 251-263, 2008.

[7] J. Idris, T. Verdel and M. Al-Heib, "Numerical modelling of masonry joints degradation in built tunnels," Tunnelling and Underground Space Technology, vol. 24, pp. 617-626, 2009.

[8] J. Mohammed, "Numerical modelling for circle tunnel under static and dynamic loads for different depth," Research Journal of Mining, vol. 1, 2017.

[9] M. A. Nunes and M. A. Meguid, "A study on the effect of overlying soil strata on the stresses developing in a tunnel lining," Tunnelling and Underground Space Technology, vol. 24, pp. 716-722, 2009.

[10] P. Jia and C. A. Tang, "Numerical study on failure mechanism of tunnel in jointed rock mass," Tunnelling and Underground Space Technology, vol. 23, pp. 500-507, 2008.

[11] P. Kumar and A. K. Shrivastava, "Various Factors Effecting Stability of Underground Structures: State of Art," $7^{\text {th }}$ Indian Rock Conference, 2017, pp. 576-580.

[12] R. Tiwari, T. Chakraborty, and V. Matsagar, "Dynamic Analysis of a Twin Tunnel in Soil Subjected to Internal Blast Loading," Indian Geotech J., vol. 46, no. 4, pp. 369-380, 2016.

[13] S. Mishra, K.S. Rao, N.K.Gupta and A.Kumar, "Damage to Shallow Tunnels under Static and Dynamic Loading," Procedia Engineering 173, 1322-1329, 2016.

[14] S. Mishra, A. Challa, A. Singh, A. Kumar, K. S. Rao and N. K. Gupta," Parametric study of lined and unlined tunnels at shallow depths under coupled static and cyclic loading conditions," Tunnelling in Himalayan Geology, vol. $340,2017$.

[15] Z. Zhu, Y. Li, J. Xie and B. Liu, "The effect of principle stress orientation on tunnel stability," Tunnelling and Underground Space Technology, vol. 49, pp. 279-286, 2015. 